\title{
Multi-Agent Base Evacuation Support System Using MANET
}

\author{
Shohei Taga*, Tomofumi Matsuzawa ${ }^{\dagger}$ and Munehiro Takimoto* \\ Department of Information Sciences \\ Tokyo University of Science \\ 2641 Yamazaki Noda 278-8510, Japan \\ *6318702@ed.tus.ac.jp \\ ${ }^{\dagger}$ t-matsu@is.noda.tus.ac.jp \\ tmune@is.noda.tus.ac.jp \\ Yasushi Kambayashi \\ Department of Computer and Information Engineering \\ Nippon Institute of Technology \\ 4-1 Gakuendai Miyashiro-machi \\ Minamisaitama-gun, Saitama 345-8510, Japan \\ yasushi@nit.ac.jp
}

Received 30 December 2018

Revised 24 February 2019

Accepted 4 March 2019

Published 29 March 2019

\begin{abstract}
In this paper, we propose an evacuation support system that provides evacuation routes in case of disasters, and verify the usefulness of the system. Current popular wireless communication infrastructure is supported by a series of base stations and one base station handles a lot of communication. Therefore, when communication base stations break down due to disasters such as an earthquake, it may become difficult for people to use their smartphones based on the Internet. When the communication infrastructure is paralyzed, people will have great difficulty collecting information about the conditions of transportation systems and about the safety of family and friends using smartphones. Our proposed system addresses this problem by using multiple kinds of mobile agents in addition to static agents on smartphones that use a mobile ad hoc network (MANET). The proposed system collects information with mobile agents and diffuses information via mobile agents so that the system is able to provide an optimized evacuation route for each user in a dynamically changing disaster environment. In this paper, we extend our previously proposed evacuation support system to consider elevation information when constructing evacuation routes. When a tsunami or a flood tide occurs, low elevations may be under water. Therefore, this revised evacuation support system
\end{abstract}

This is an Open Access article published by World Scientific Publishing Company. It is distributed under the terms of the Creative Commons Attribution 4.0 (CC-BY) License. Further distribution of this work is permitted, provided the original work is properly cited. 
helps people to move to safer places by selecting higher elevation routes when warranted by the situation.

Keywords: Mobile ad hoc network; mobile agent; multi-agent; contingency plan; risk management.

\section{Introduction}

In this paper, we propose an evacuation support system that develops optimized evacuation routes in the event of a disaster, and we verify the usefulness of the system. In the recent years, with the development of communication and portable device technologies, people can collect and spread information using the Internet regardless of time and place. Current popular wireless communication infrastructure is supported by a series of base stations; components of this infrastructure, such as a base station, handle a lot of communication. Therefore, when problems occur, it may be difficult, or impossible, for the smartphones to use the Internet. In the 2011 Tohoku Earthquake off the Pacific coast of Japan, we observed a largescale communication failure due to corruption of the communication equipment and traffic congestion. Paralysis of the communication infrastructure made it difficult for people to collect information using smartphones about the conditions of transportation infrastructure and about the safety information of family and friends.

Our proposed system addresses this problem of communication infrastructure by constructing mobile ad hoc network (MANET) by wireless communication between users' smartphones enabling users to share information via such a network. Since MANET is a network constructed with only portable devices, it is possible to avoid problems due to failure of centralized communication infrastructure. The ever changing topology of MANET, however, makes stable communication extremely difficult. Therefore, we propose an information sharing method using mobile software agents. A mobile agent is a program with mobility; it is able to sense the state of its environment and can alter its behavior accordingly. Our proposed system constructs optimized evacuation routes using such mobile agents to share and collect information necessary for evacuation.

In the previous studies, we proposed the basic configuration of such an evacuation support system and verified its feasibility with simulation. ${ }^{1,2}$ In this study, we have expanded the features of the system and verify its usefulness with a simulator named NS-3. In the previous papers, we only employed mobile agents to disseminate information about the circumstances. The evacuation users, therefore, received information not necessarily related to their potential evacuation routes, while having not receiving crucial information for their evacuation (i.e. potential dangers on their evacuation routes). In order to improve the system, in this paper, we introduce another kind of mobile agent that actively collects information closely related to the users' potential situation. 
NS-3 is a discrete-event network simulator which was designed primarily for research and educational use. ${ }^{3}$ The previous simulators were crude and did not reflect the real MANET situation. On the other hand, NS-3 models the communication environments and processes in a form close to the real world, and makes it possible to perform various verification experiments. Using NS-3 makes an accurate simulation of an ad hoc network feasible.

This paper is an extended version of the work originally presented in the Tenth International Conference on Computational Collective Intelligence. ${ }^{4}$ In addition to the previous paper, we consider altitude information for constructing evacuation routes in this paper. The extension portion was partially reported in the Eleventh International Conference on Agents and Artificial Intelligence. ${ }^{5}$ If a tsunami or flood tide occurs, evacuating through low elevation routes is very dangerous. In order to guide people to evacuate safely, evacuees should be directed to move along higher elevation routes. Therefore, we propose a new algorithm that is able to construct evacuation routes that can include altitude data, and extend our simulator to verify the algorithm.

The structure of this paper is as follows: Sec. 2 describes the related works. Section 3 describes the details of the proposed system. Section 4 describes the numerical experiments and discusses the results, and Sec. 5 discusses future works and concludes the discussion.

\section{Related Works}

Komatsu et al. proposed an evacuation system that estimates impassable points automatically by comparing evacuation route presented by the system and actual evacuees' trajectories. ${ }^{6}$ Estimated impassable points are shared between evacuees by communication between their portable devices or server through available communication infrastructure.

Wang et al. proposed a solution for fire evacuation routing problems by applying artificial bee colony optimization (BCO) algorithm. ${ }^{7}$ The BCO algorithm is swarm intelligence algorithm inspired by the foraging behavior of bees. They simulated this solution for evacuation from buildings with multiple exits to improve the total evacuation time.

Ikeda et al. proposed the safety evacuation route planning using multi-objective genetic algorithm (MOGA). ${ }^{8}$ The MOGA is a method applying genetic algorithm to the multi-objective optimization problem. An evacuee's handset records GPS data and acceleration data, and sends them to a crowd server. The server calculates an evacuation route considering evacuation distance, evacuation time and the safety degree of evacuation route, and then provides the optimal route to evacuees.

Kartalidis et al. proposed an evacuation routing method that detects the position of the evacuee by using trilateration using wireless access points and the evacuee's smartphone, and then constructs an evacuation route based on cellular automata. ${ }^{9}$ The states of cellular automata express the state of specific positions within the 
evacuation area, indicating the presence of evacuees detected, obstacles, free area and more. For each step of the exit path exploration process, the state of each cell is refreshed. Therefore, evacuees can evacuate avoiding impassable points.

Asakura et al. proposed an algorithm to calculate a simple evacuation route to reduce the difficulty for evacuees. ${ }^{10}$ If the evacuation route is complicated and meandering, the evacuees may be confused. In order to mitigate this problem, their method calculates a route that has the fewest number of turns at intersections.

Avilés et al. proposed an evacuation support system using MANET and Ant Colony Optimization (ACO) for indoor environments. ${ }^{11}$ In the study, they implemented ACO by using mobile agents so that the ACO algorithm can take the movement trajectory and speed of evacuees into consideration. Then the evacuation support system constructs an optimal evacuation route for each user.

Ohta et al. also considered the evacuation support methods using ACO and MANET. ${ }^{12}$ In their study, they pointed out the problem that a conventional ACO may include dangerous locations when it constructs evacuation routes. This problem is caused by the dynamic nature of the disaster environments such as conflagration or tsunami. In order to mitigate this problem, they proposed an improved ACO-based evacuation support system that adds a deodorant pheromone which erases ACO pheromone traces when dangerous locations are found. Goto et al. applied this proposed method to real data of tsunami damage. ${ }^{13}$ They showed practical results by using the data of Rikuzentakata city which suffered great damage due to the 2011 Tohoku Earthquake. They verified the method of Ohta et al. which is feasible based on the real data.

Kambayashi et al. proposed and implemented a system that collects safety information of evacuees using mobile agents and MANET. ${ }^{14}$ In the study, they proposed a method to reduce load on transmission by combining multiple mobile agents into one. Nishiyama et al. proposed a communication system using portable devices that switch between MANET and Delay Tolerant Networking (DTN) according to the communication situation. ${ }^{15}$ DTN is a method for coping with a network environment where maintaining stable communication connection is hard to achieve. When communication is disconnected, portable devices accumulate data, and then transmitted when communication is resumed. Their proposed system applies MANET when there are many portable devices in the surroundings, and applies DTN when there are few, to cope with changing network environments.

\section{Agent Base Evacuation Support System}

In this section, we describe our proposed system in detail. The proposed system aims to provide an optimal evacuation route for each user (whom we call the evacuation user). Since the proposed system maintains the map information of the evacuation area, it is possible to calculate the shortest route to the destination (i.e. safe place). However, at the time of a disaster, there are likely to be many occurrences of unsuitable points for evacuation (hereafter we call dangerous point) due to fire, building 
collapse or inundation. Since nobody knows these points before the occurrence of a disaster, it is necessary to collect the information during evacuation. When an evacuation user finds a dangerous point, he or she inputs the position information into the system. We assume that the smartphone can connect to the satellite network to record GPS data. Then the proposed system constructs a new evacuation route avoiding this dangerous point, and provides it to the user. At the same time, the system diffuses the information about the dangerous point and new route to other users' smartphones. As a result, evacuation users other than the discoverer can know the dangerous point and avoid it in advance. In order to realize this function, we use multiple mobile agents.

A multi-agent system is a system that consists of multiple agents and achieves tasks by their cooperative operations. The agents can be categorized into two types: mobile agents and static agents. A mobile agent is generated when it is needed and executes a task by migrating among communication sites including smartphones. Every mobile agent has a unique identifier. A static agent resides on each communication site including, of course, a smartphone. Unlike mobile agents, a static agent has no unique identifier. We describe the details as follows.

\subsection{Static agents}

\subsubsection{Information agent}

Information Agent is a static agent residing on a smartphone that interacts with mobile agents and constructs evacuation routes. When requested from the user, it creates mobile agents.

The information agent processes the request in the following order: (i) It generates the requested mobile agent. (ii) It acquires the information necessary for the generated mobile agent from the node management agent and passes it to the mobile agent. (iii) It stores the mobile agent in a queue. It periodically checks the queue, and dispatches the mobile agents to the neighboring smartphones. When a mobile agent comes from another smartphone, the information agent receives information held by the mobile agent. Then it passes the requested information to the arrived mobile agent and store it in the queue in the same way as (iii). The information agent records the unique identifier of the mobile agent that visited the smartphone as well as it created in a list called visitor list. The information agent requests the visitor list of other smartphones when it communicates with them. It then passes the received visitor list to the mobile agent that needs it in the queue. The mobile agent decides the next destination from this visitor list.

The information agent constructs evacuation routes based on the information it initially has, and the information collected from the arriving mobile agents. The evacuation route is the route to the destination avoiding dangerous points that are currently known by the information agent. The evacuation route is determined based on the Dijkstra's algorithm. The Dijkstra's algorithm is an algorithm for solving the shortest path problem between two nodes in a graph, and was proposed 
by Dijkstra in $1959 .{ }^{16}$ In the proposed system, the graph consists of intersections as the nodes, and the distances between intersections as the edge weights. In this proposed system, in order to construct higher elevation evacuation routes, the system calculates edge weight by dividing distance by the altitude of the node. Hence, a high altitude route is preferentially selected as evacuation route. This evacuation route may not be the shortest route, but evacuation user can avoid a tsunami or flood tide and reach safe areas. The information agent constructs an initial evacuation route at the system startup time. After that, when a mobile agent arrives and let the information agent know a dangerous point exists on the current evacuation route, the information agent reconstructs the evacuation route.

\subsubsection{Node management agent}

Node Management Agent is another static agent residing on a smartphone for managing the information on the smartphone. The node management agent stores the dangerous point information collected from the mobile agents that have arrived in the information table. At this time, if the same information already exists in the information table, the node management agent deletes older information. Also, if the information agent requests information about dangerous points, the node management agent passes the requested information.

\subsection{Mobile agents}

\subsubsection{Information diffusion agent}

Information Diffusion Agent is a mobile agent that diffuses the information of the dangerous point found by the evacuation user to neighboring smartphones.

When an evacuation user finds a dangerous point and inputs its information into the system, the information agent generates the information diffusion agent. The information agent passes the coordinates of the discovered point to the generated information diffusion agent. And then, the information diffusion agent waits until a link with another smartphone is established. After that the information diffusion agent acts as follows: (i) When communication links with other smartphones are established, the information diffusion agent copies itself by the number of linked smartphones and moves to each smartphone. However, if the information diffusion agent finds that it has already visited the smartphone (i.e. the smartphone's visitor list has its identifier), it does not move but commits suicide. (ii) After arriving at a new smartphone, it passes its own information to the information agent at the destination, and goes into standby state until the next communication link is established. It repeats this process a constant number of hops. When the information diffusion agent copies itself, it copies not only its own information but also its own unique identifier. Therefore, it does not move to a smartphone that its own copy has visited. This mechanism prevents a smartphone from receiving the same information multiple times. 


\subsubsection{Information collecting agent}

Information Collecting Agent is another mobile agent that collects information about the events on the evacuation route and returns to the original smartphone. The information diffusion agent diffuses the information discovered by the evacuation user around the discovery point, but there is no guarantee that this information can be conveyed to all evacuation users who need it. In order to solve this problem, we propose an information collecting agent that actively collects information diffused by the information diffusion agents as shown in Fig. 1.

The information collecting agent has two states, the collecting state and the return state, and its behavior changes depending on the state. Initially, it is in the collecting state. In the collecting state, it acts as follows: (i) The information collecting agent moves to the smartphones along the current evacuation route of the user. It excludes smartphones that it has already visited. (ii) It acquires information from the destination smartphone. The information collecting agent repeats this process during the collecting state. If the information collecting agent acquires information that tells it that there is a dangerous point on the evacuation route or if it has exceeded half of its own life time or cannot find the next migration candidate, the information collecting agent transitions to its return state in which it attempts to return to its originating smartphone. There are, however, problems to be overcome to determine the return route since it is necessary to handle unexpected environmental obstacles to the safety of the evacuation users. Therefore, we cannot assume that the information collecting agent will be able to return to the originating evacuation user using the movement history of the agent in reverse order since the smartphones it had visited on its route may since have disappeared or become inaccessible. Those smartphones may have moved out of wireless communication range or their batteries may be exhausted. Therefore, this information collecting agent waits until a communication link with another smartphone is established. In its return state, it acts as follows: (i) The information collecting agent calculates the predicted current position of the original

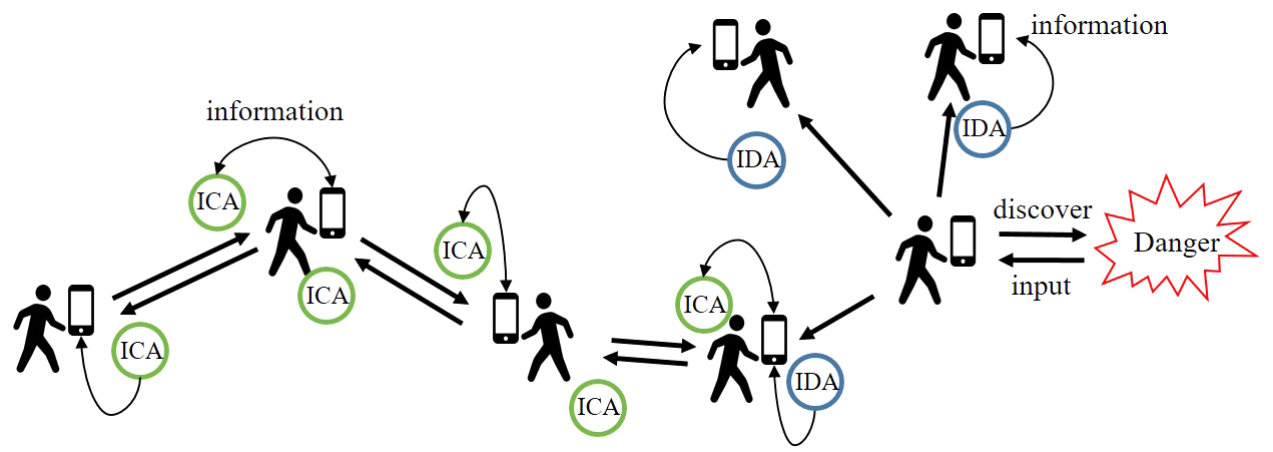

Fig. 1. Information diffusion and information collection. ICA means the information collecting agent. IDA means the information diffusion agent. 


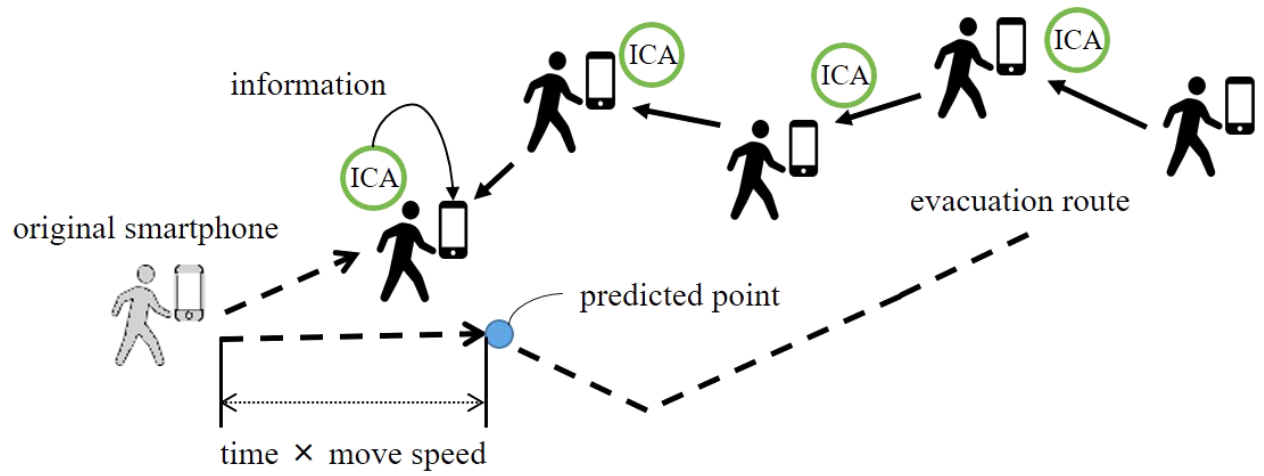

Fig. 2. Predict the position of original smartphone.

smartphone based on the information it has; i.e. evacuation route information of the original smartphone and the moving speed of the evacuation user of the smartphone, and the elapsed time since it was generated as shown in Fig. 2. (ii) It moves to the still accessible smartphones in its history lists that are closest to the calculated expected position.

Unlike the collecting state, in the return state, it may move to a smartphone it has visited before. It repeats this process until returning to the original smartphone. When the information collecting agent returns to the original smartphone, it passes the collected information to the information agent on the smartphone and disappears. If the information collecting agent cannot find the original smartphone in the vicinity of the calculated predicted current position, within its defined life time, it commits suicide.

One of the disadvantages of the information collecting agent is that, since it is generated from each smartphone, the network load tends to increase. In order to mitigate this problem, the proposed system controls the generation of the information collecting agents by broadcasting messages to the neighboring smartphones that request them to stop generating information collecting agents for certain period. This message contains the address and the evacuation route of the sender, and the life time of the information collecting agent. A smartphone that receives this message stops generating information collecting agents if its own evacuation route is the same as described in the message. If its own evacuation route is different from what described in the message, it ignores and discards the message. When this information collecting agent returns to the sender smartphone of the stop message, that smartphone broadcasts messages that permit generation of information collecting agents and also the collected information to the neighboring smartphones. The smartphones that have stopped generating information collecting agent resume generation of the agents when they receive this message. The smartphones also resume generating information collecting agents when the stop period in the message elapses. 


\section{Numerical Experiments}

This section describes the simulation testing of the proposed system. We verified our system by simulation of situations in which people use the proposed system during a disaster. We used NS-3 for simulation. NS-3 is a discrete-event network simulator which is open for research and educational use. ${ }^{3}$ We are able to model the communication environments and processes in a form close to the real world, and the simulator makes it possible to evaluate the performance of our system under various scenarios.

\subsection{Experimental conditions}

In order to model two different disaster scenarios in the same geographic area, we created two simulation maps that represent the evacuation areas (Fig. 3). Both simulation maps show a part of downtown Tokyo, and are modeled on the real geography. These maps consist of nodes and edges. The edge is a straight sidewalk, where evacuees move along the edge. A node is an intersection point of edges. For the destinations of evacuees, several safe areas are provided in the map. Map (a) represents serious fire involving a large area. This map is about one square kilometer in the real map, and does not include elevation information. There are 266 nodes and four safe areas. Map (b) represents a major flood. This map represents about $4.5 \mathrm{~km}^{2}$ of the real map and includes elevation information. There are 1,430 nodes and five safe areas. The lowest altitude node is $5.4 \mathrm{~m}$ and the highest altitude node is $32.0 \mathrm{~m}$.

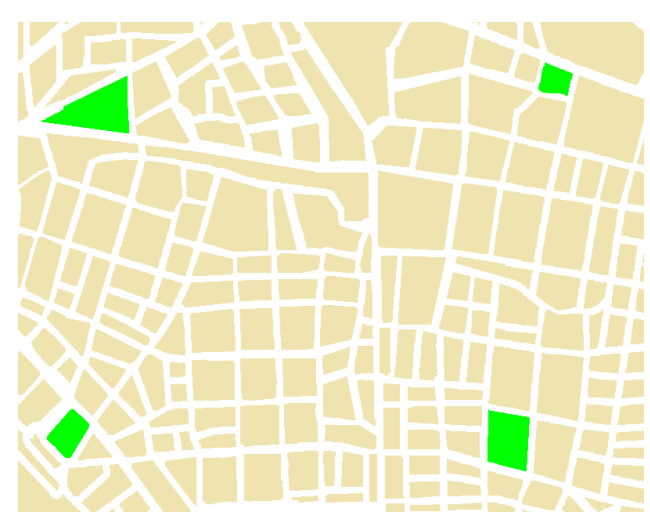

(a)

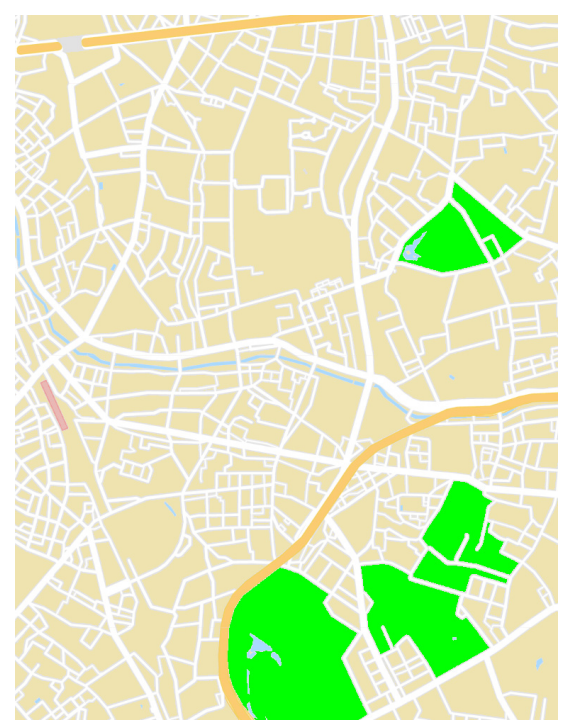

(b)

Fig. 3. Simulation maps: Map (a) represents a major fire. Map (b) is used for the simulation of major flooding such as a tsunami or flood tide. 
Evacuation users are randomly placed and move toward the nearest safe area from their current positions. The moving speed of the evacuation user is set to $1 \mathrm{~m} / \mathrm{s}$. The proposed system has the map information. Each evacuee moves along the evacuation route constructed by the information agent in his or her smartphone. The proposed system does not have dangerous point information in advance, and those points will become known to an evacuation user for the first time when that evacuation user actually touches the dangerous point or is notified by other evacuation users through mobile agents. When the information agent of an evacuation user learns of a new dangerous point, the information agent reconstructs the evacuation route to avoid this dangerous point. At this time, the previous nearest safe area from current position of evacuation user may change. In such a case, the information agent changes the destination to a new nearest safe area. The dangerous points are gradually increased on the user's map as the simulation progresses. How dangerous points are created during the simulation differs between the two maps. In Map (a), a random node becomes dangerous point, since flying sparks can start new fires in unexpected quarters. In Map (b), in order to simulate a flood tide disaster, nodes become dangerous points from the lowest altitude node, i.e. along the river. In both maps, the dangerous points increase every $10 \mathrm{~s}$ (Fig. 4). The communication distance of the smartphone is $50 \mathrm{~m}$. Smartphone communication and evacuation user movement stops in this simulation for the evacuation user who arrives at a safe area (hereafter called a safe evacuation user), or one who arrives at a location surrounded by dangerous points from which it is impossible to arrive at a safe area (hereafter called a dead evacuation user). When all evacuation users stop, the simulation ends.

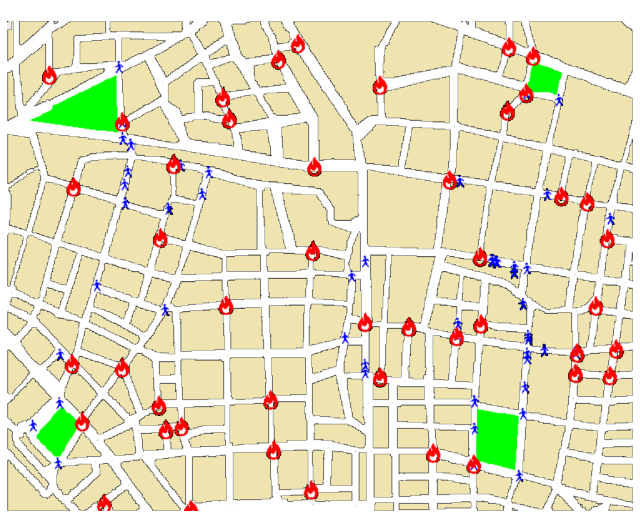

(a)

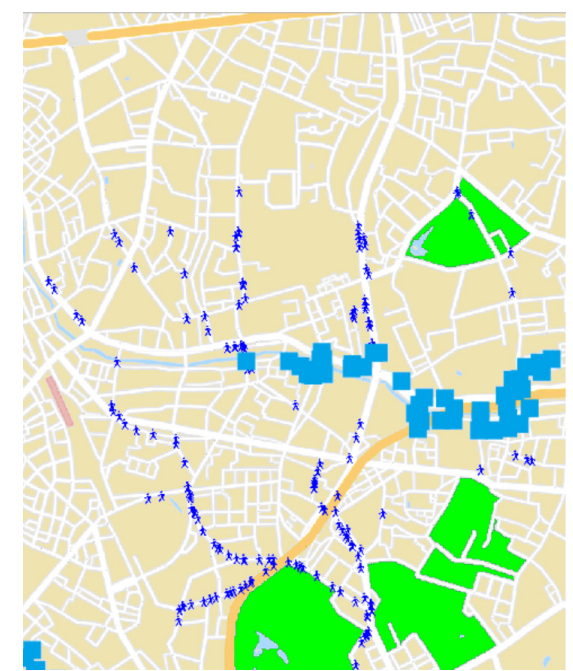

(b)

Fig. 4. Simulation maps with dangerous points and evacuees. The dangerous points are flame icons on Map (a) and square icons on Map (b). The evacuees are walker icons. 
In this verification experiment, we measured (1) the number of the touches to dangerous points by all evacuees, (2) the number of the safe evacuation users and dead evacuation users. We performed the simulation for both Maps (a) and (b). In the case of using Map (a), we compared outcomes in the case of using the proposed system and in the case of not using it (i.e. evacuation user's smartphone calculates an evacuation route to safe area, but does not share information about dangerous points). In the case of using Map (b), in addition to the above two cases, in the case of using the proposed system, we verified outcomes in the cases of sharing dangerous points with and without elevation information, and of not sharing information about dangerous points found (and omitting elevation information).

In our simulation runs, the number of hops of an information diffusion agent is 1 , and the life time and the generation interval of an information collecting agent is set to $120 \mathrm{~s}$. For all scenarios, we evaluated the system with varying numbers of evacuees: 50, 100, 150 and 200. We have carried each scenario 50 times and taken the averages as the results.

\subsection{Results and discussion}

First, we describe the experimental results for Map (a). Figure 5 shows the results of the number of the touches to the dangerous points. In this figure, "use" is the case of using the proposed system and "not use" is the case of not using it. In all the results, we found that in the case of using the proposed system suppressed the number of touches to the dangerous points better than in the case of not using it.

Figure 6 shows the results of the number of the safe evacuation users and the dead evacuation users. In this figure, "Safe" is the safe evacuation user and "Dead" is the dead evacuation user where the number of evacuees is 50 and 100, there is no significant difference in the results. On the other hand, in the cases of the number of evacuees is 150 and 200, the "use" is more useful than the "not use". If the evacuation users who participate the MANET are a few, evacuees can only know information about nearby dangers because the network is only partially constructed.

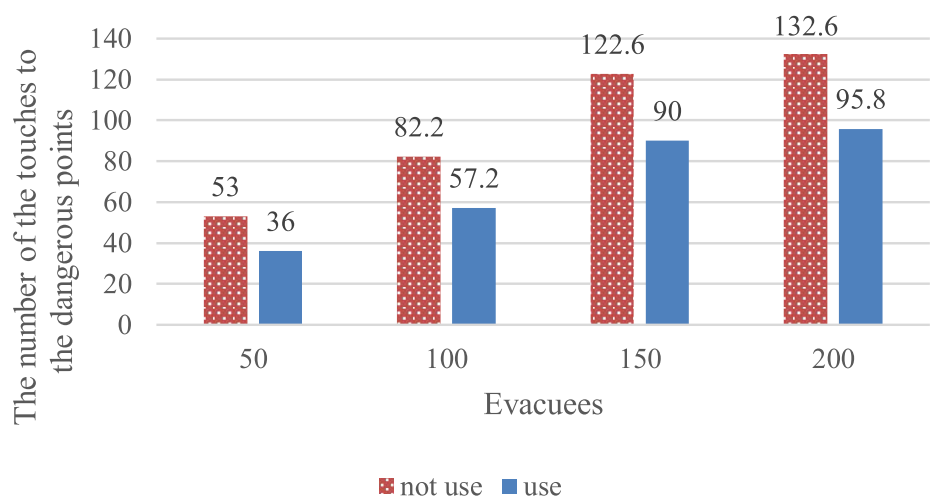

Fig. 5. Results of the number of the touches to the dangerous points in the case of using Map (a). 


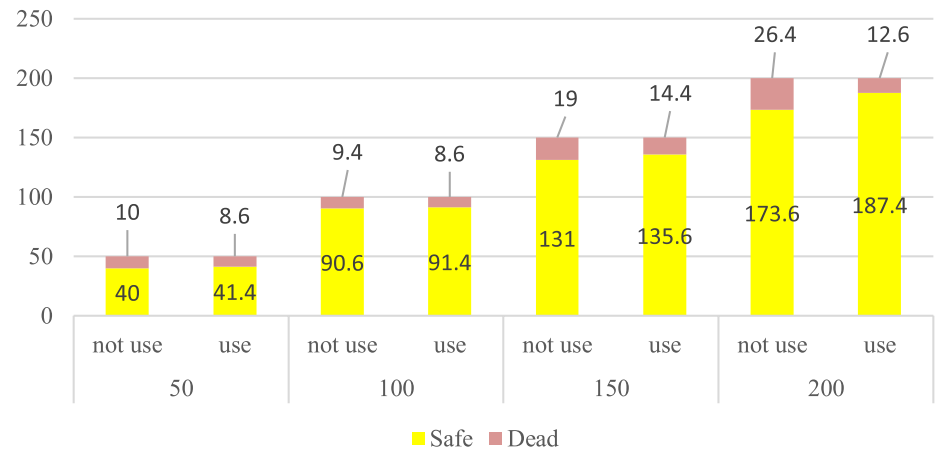

Fig. 6. Results of the number of the safe evacuation users and the dead evacuation users in the case of using Map (a).

Therefore, an evacuee near to a dangerous point may be surrounded by other dangerous points before escaping, even if he or she could know the information of that point in advance. On the other hand, if there are many evacuation users, they can learn of situations in distant area, and they can escape from dangerous areas before being surrounded by the dangerous points.

Next, we describe the experimental results for Map (b). Figure 7 shows the results of the number of the touches to the dangerous points. "no altitude" is the case of not considering altitude and "altitude" is the case of considering altitude. "not use" is the same as in Map (a). In the case of the number of the evacuees is 50-150, there is no significant difference between the "not use" and the "no altitude", but in the case of the number of evacuees is 200 , the "not altitude" is smaller than the "no use". In all cases, the "altitude" shows good results.

Figure 8 shows the results of the number of safe evacuation users and dead evacuation users. Unlike the results shown in Fig. 7, where the number of the evacuees is 200 , there is no significant difference between the results for "no altitude" and "not use". On the other hand, "altitude" shows good results in all the cases. As mentioned before, the proposed system is more effective if the number of the evacuees

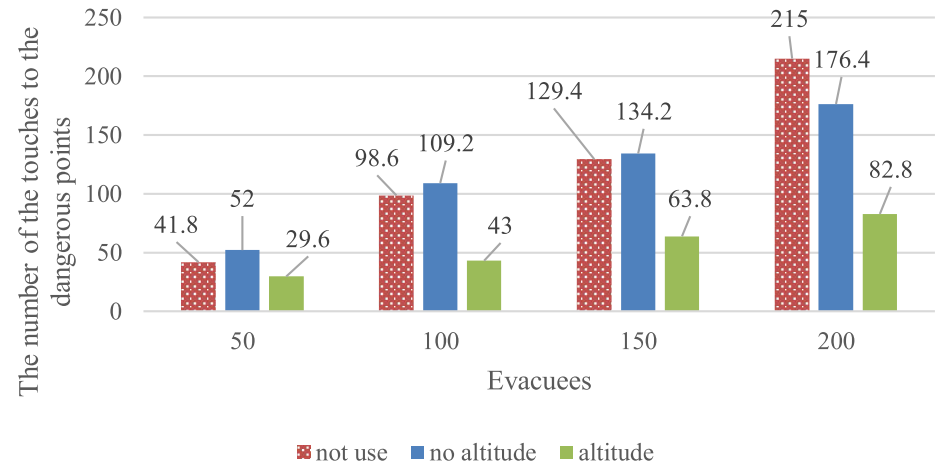

Fig. 7. Results of the number of the touches to the dangerous points in the case of using Map (b). 


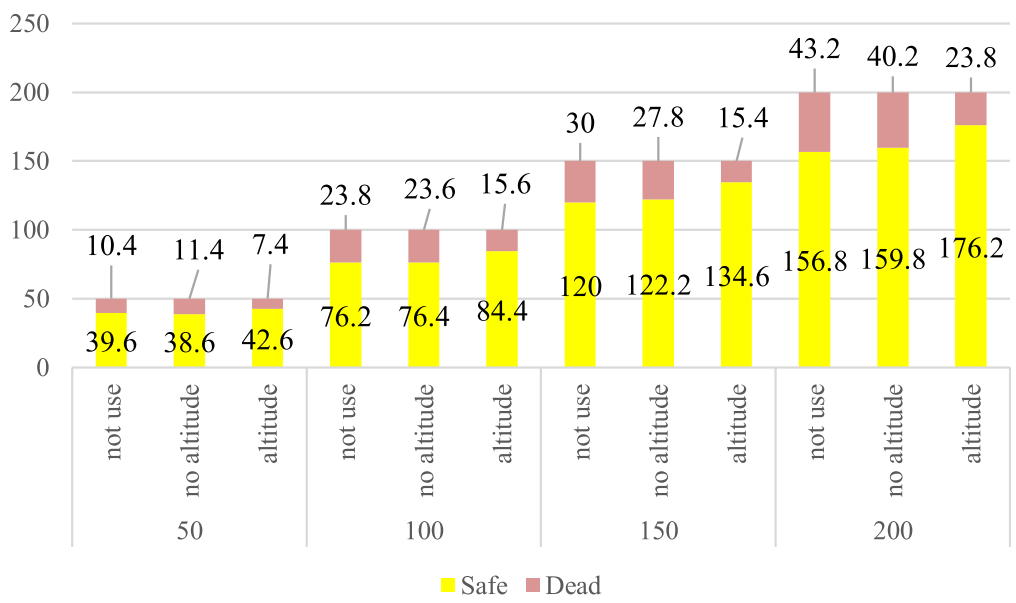

Fig. 8. Results of the number of the safe evacuation users and the dead evacuation users in the case of using Map (b).

is large. As Map (b) is wider than Map (a), the number of evacuees has more influence. Evacuees close to dangerous points, however, have the danger of being surrounded by other dangerous points even if they obtain information of the points and avoid them. In particular, in Map (b), as it submerges from the lowest place, an evacuee close to a dangerous point is surrounded immediately by the other dangerous points. Therefore, selecting higher evacuation routes by avoiding places with low altitude in advance contributes to evacuees' safe evacuation.

\section{Conclusion}

In this paper, we proposed an evacuation support system that enables information sharing under environments where people cannot use Internet communication due to natural disasters. In the system, we also took account of the altitude information when constructing evacuation routes. In the experimental section, we showed that evacuees can be informed about dangerous points in advance and avoid them by using the proposed system. Although the current mobile agents collect dangerous point information, they may carry information about safe points such as the first aid stations. We need to investigate how much communication traffic increases for such cases. We also showed that evacuees can safely evacuate by choosing high altitude routes as evacuation routes when they are hit by tsunami and flood tides. Actually, tsunami and river flooding have different characteristics. We need further investigation.

As a future work, it is necessary to address the increase of the load in the relay smartphones. Since the information collecting agent moves along the evacuation route of the evacuation user, they frequently move in a busy street such as main streets and in front of evacuation centers. As a result, the network load drastically 
increases in particular places. Therefore, it is necessary to develop a mechanism for controlling the amount of communication traffic caused by the agent in such places. In addition, we need to consider the length of the evacuation time when choosing high altitude route. By preferentially selecting high altitude routes, evacuees can safely escape from flood waters. Since this route is not the shortest route to the safe area, however, evacuation time will increase. In order to solve this problem, it is conceivable to change the priority of selecting a route with a high altitude according to the altitude of the current position of the evacuee. If the current position of the evacuee is a place with a low altitude, it is necessary to move quickly to a high place. But if evacuee is in a sufficiently high place from the beginning, the necessity of selecting a higher place is low. In this case, it may be safer to move a route with shorter distance than to move a route with a longer but higher altitude.

\section{Acknowledgments}

The authors appreciate Kimiko Gosney for her contribution in the completion of this paper. She gave us useful comments. This work is partially supported by Japan Society for Promotion of Science (JSPS), with the basic research program (C) (Nos. 17K01304 and 17K01342), Grant-in-Aid for Scientific Research (KAKENHI).

\section{References}

1. S. Taga, T. Matsuzawa, M. Takimoto and Y. Kambayashi, Multi-agent approach for return route support system simulation, in Proc. 8th Int. Conf. Agents and Artificial Intelligence, Vol. 1, Rome Italy (Science and Technology Publications, Lda., Setubal, Portugal, 2016), pp. 269-274.

2. S. Taga, T. Matsuzawa, M. Takimoto and Y. Kambayashi, Multi-agent approach for evacuation support system, in Proc. 9th Int. Conf. Agents and Artificial Intelligence, Vol. 1, Porto, Portugal (Science and Technology Publications, Lda., Setubal, Portugal, 2017), pp. 220-227.

3. Nsnam, ns-3 - a discrete-event network simulator for internet systems, https://www. nsnam.org/, last accessed March 15, 2019.

4. S. Taga, T. Matsuzawa, M. Takimoto and Y. Kambayashi, Multi-agent base evacuation support system using MANET, in 10th Int. Conf. Computational Collective Intelligence, Part 1, Bristol, UK, LNCS 11055 (Springer Nature Switzerland AG, Cham, Switzerland, 2018), pp. 445-454.

5. S. Taga, T. Matsuzawa, M. Takimoto and Y. Kambayashi, Multi-agent approach for evacuation support system considering altitude, in Proc. 11th Int. Conf. Agents and Artificial Intelligence, Vol. 1, Prague, Czech Republic (Science and Technology Publications, Lda., Setubal, Portugal, 2019), pp. 299-306.

6. N. Komatsu, M. Sasabe, J. Kawahara and S. Kasahara, Automatic evacuation guiding scheme based on implicit interactions between evacuees and their mobile nodes, GeoInformatica 22(1) (2018) 127-141.

7. C. Wang, L. C. Wood, H. Li, Z. Aw and A. Keshavarzsaleh, Applied artificial bee colony optimization algorithm in fire evacuation routing system, J. Appl. Math. 2018 (2018) 7962952 . 
8. Y. Ikeda and M. Inoue, An evacuation route planning for safety route guidance system after natural disaster using multi-objective genetic algorithm, Procedia Comput. Sci. 96 (2016) 1323-1331.

9. N. Kartalidis, I. G. Georgoudas and G. C. Sirakoulis, Cellular automata based evacuation process triggered by indoors Wi-Fi and GPS established detection, in 13th Int. Conf. Cellular Automata for Research and Industry (Springer, Cham, 2018), pp. 492-502.

10. K. Asakura and T. Watanabe, An algorithm for calculating simple evacuation routes in evacuation guidance systems, in Intelligent Interactive Multimedia Systems and Services (Springer, Cham, 2016), pp. 287-295.

11. A. Avilés, M. Takimoto and Y. Kambayashi, Distributed evacuation route planning using mobile agents, Transaction on Computational, Collective Intelligence XVII, LNCS 8790 (Springer, Berlin, Heidelberg, 2014), pp. 128-144.

12. A. Ohta, H. Goto, T. Matsuzawa, M. Takimoto, Y. Kambayashi and M. Takeda, An improved evacuation guidance system based on ant colony optimization, Intell. Evol. Syst. 5 (2015) 15-27.

13. A. Ohta, H. Goto, T. Matsuzawa, M. Takimoto, Y. Kambayashi and M. Takeda, A guidance system for wide-area complex disaster evacuation based on ant colony optimization, in Proc. 8th Int. Conf. Agents and Artificial Intelligence, Vol. 1, Rome Italy, (Science and Technology Publications, Lda., Setubal, Portugal, 2016), pp. 262-268.

14. Y. Kambayashi, T. Nishiyama, T. Matsuzawa and M. Takimoto, An implementation of an ad hoc mobile multi-agent system for a safety information, in Proc. 36th Int. Conf. Information Systems Architecture and Technology - ISA T 2015 - Part II (Springer, Cham, 2015), pp. 201-213.

15. H. Nishiyama, M. Ito and N. Kato, Relay-by-smartphone: Realizing multi-hop device-todevice communications, IEEE Commun. Mag. 52(4) (2014) 56-65.

16. E. W. Dijkstra, A note on two problems in connexion with graphs, Numer. Math. 1 (1959) 269-271. 